\title{
Nano-granular texture of cement hydrates
}

\author{
Katerina loannidou ${ }^{1,2, \star}$, Franz-Josef Ulm² ${ }^{2}$ Pierre Levitz ${ }^{3}$, Emanuela Del Gado ${ }^{4}$, and Roland J.-M. Pellenq ${ }^{1,2,5, \star \star ~}$ \\ ${ }^{1}$ Multiscale Material Science for Energy and Environment, MIT - CNRS Joint Laboratory at Massachusetts Institute of Technology, \\ Cambridge, Massachusetts 02139, USA \\ ${ }^{2}$ Department of Civil and Environmental Engineering, Massachusetts Institute of Technology, Cambridge, Massachusetts 02139 , \\ USA \\ ${ }^{3}$ PHENIX Laboratory, CNRS and University Pierre et Marie Curie, 75252 PARIS cedex 5, France \\ ${ }^{4}$ Department of Physics and Institute for Soft Matter Synthesis and Metrology, Georgetown University, Washington, DC 20057, \\ USA \\ ${ }^{5}$ CINaM, CNRS and Aix-Marseille University, Campus de Luminy, 13288 Marseille Cedex 09, France
}

\begin{abstract}
Mechanical behavior of concrete crucially depends on cement hydrates, the "glue" of cement. The design of high performance and more environmentally friendly cements demands a deeper understanding of the formation of the multiscale structure of cement hydrates, when they precipitate and densify. We investigate the precipitation and setting of nano-grains of cement hydrates using a combination of Monte Carlo and Molecular Dynamics numerical simulations and study their texture from nano up to the micron scale. We characterize the texture of cement hydrates using the local volume fraction distribution, the pore size distribution, the scattering intensity and the chord length distribution and we compare them with experiments. Our nano-granular model provides cement structure with realistic texture and mechanics and can be further used to investigate degradation mechanisms.
\end{abstract}

\section{Introduction}

Concrete is the most used manufactured material as it is the cheapest and durable, and has outstanding mechanical performances. Cement is the binder of concrete and calcium-silicate-hydrates (C-S-H) is the main hydration product of cement which acts as the glue of the material. Understanding the formation of the multiscale structure of C-S-H is important for controlling the macroscopic properties of cement upon chemical modifications.

When water is mixed with cement powder, cement begins to dissolve. Upon saturation of the ionic solution, $\mathrm{C}-\mathrm{S}-\mathrm{H}$ precipitates in the form of nano-grains. C-S-H is a nonstoichiometric hydration product with calcium to silicon $(\mathrm{C} / \mathrm{S})$ ratio ranging from 1 to 2.2 depending on the composition of the cement powder [1]. The inner structure of C-S-H nano-grains is complex, resembling that of Tobermorite minerals at low $\mathrm{C} / \mathrm{S}$ and that of disordered glasses at high C/S. Recent advances of atomistic modeling of C-S-H have revealed characteristic of the structure and mechanical properties of the nano-grains [2]. Moreover they have provided a first description of the interaction potentials between such grains. However cement is a multiscale material and its macrocopic mechanical response relies not only on the chemistry of the nano-grains but also on the mesoscale structure that they form upon aggregation.

\footnotetext{
^e-mail: hekate@mit.edu

$\star \star$ e-mail: pellenq@mit.edu
}

The setting, during which cement is transformed from a liquid paste to a soft and finally hard solid, is intimately connected to the hydration process, when $\mathrm{C}-\mathrm{S}-\mathrm{H}$ grains precipitate [3]. The complicated evolution with time indicates that $\mathrm{C}-\mathrm{S}-\mathrm{H}$ can have very different cohesion and space filling properties, not only due to initially different chemical composition but also due to the interplay between aggregation and arrested kinetics through the hydration and setting process $[4,5]$.

Here, we present a recent model for $\mathrm{C}-\mathrm{S}-\mathrm{H}$ grains precipitation and aggregation that is based on the potentials of mean field extracted at the atomistic scale and accounts for the structure and the mechanical response of the material all the way up to the micron scale. We characterize the nano-granular structure in terms of local volume fractions, pore size distribution, scattering intensity and chord length distributions.

\section{Methods}

\subsection{Nano-granular model for cement hydrates}

A cement hydrate is described as a spherical nano-grain of polydisperse size between 3.78 to $9.2 \mathrm{~nm}$. These units (grains) have been reported in Small Angle Neutron Scattering (SANS) experiments [6, 7]. The nano-grains interact with short range attractive potential, prototypical of cement hydrates [8]. Recently, a group of such interaction potential for cement hydration has been investigated [5]. 
Here, we investigate cement at late stages of cement hydration when the effective interaction is purely attractive and described with a generalized Lennard-Jones potential

$$
V(r)=6 \epsilon\left[\left(\frac{\sigma}{r}\right)^{2 \gamma}-\left(\frac{\sigma}{r}\right)^{\gamma}\right],
$$

where $r$ is the inter-particle distance, $\epsilon$ is the well depth between two particles with diameter $\sigma$ and we have fixed the exponent to $\gamma=12$ [4].

The precipitation of nano-grains during cement hydration is modelled by a combination of Molecular Dynamics with Grand Canonical Monte Carlo (GCMC) simulations. GCMC accounts for the interplay between the chemistry, in specific the free energy gain associated to the production of one new cement hydrate, and the attractive interactions between the nanograins. This mechanism drives the aggregation of grains and densification of the system. This non-equilibrium scheme can capture several features of the kinetics of cement hydration [4, 9].

A GCMC cycle consists of $N_{M C}$ attempts of particle insertion or deletion followed by $N_{M D}=100 \mathrm{MD}$ steps in NVT ensemble. $R=N_{M C} /\left(N_{M D} \cdot L^{3} \cdot \delta t\right)$ where $L$ is the length of the simulation box and $\delta t=0.0025 \sqrt{m \sigma^{2} / \epsilon}$, is the rate of hydrate production and in this work was $R=25 \cdot 10^{-9} \delta t^{-1} \mathrm{~nm}^{-3}$. Simulations with higher values of $\mathrm{R}$ bring the system further out of equilibrium [4].Time was measured by MD unit time $\sqrt{m \sigma^{2} / \epsilon}$, the reduced temperature was $T=0.15$ and the chemical potential $\mu=-1$ in Lennard-Jones units. The chemical potential is chosen to promote densification [5]. The simulations have been performed in box size $585.54 \mathrm{~nm}$ with particles up to 610000 .

Averages have been performed over at least 3 independently generated samples and for the error bars of the results shown we used the standard deviation of the sampleto-sample fluctuations. The error bars are equal to the symbol size.

\subsection{Characterization of the texture}

The mesoscale structure obtained from the precipitation simulations is characterized using local volume fractions, the pore size distribution, scattering intensity and the chord length distribution.

The local volume fraction $\eta_{\text {local }}$ is the volume occupied in the neighborhood of one cement hydrate and is computed in a spherical region of radius $35 \mathrm{~nm}$ around each spherical grain. The radius of a grain is defined by the equilibrium distance of two grains $r_{i}=\sqrt[12]{2} \sigma_{i}$ from Eq.1.

The pore size distribution is computed using the technique of Ref. [10] by the insertion of a probe sphere in a point $k$ of the pore. The size of the pore at point $k$ is the diameter $R$ of the largest sphere that contains $k$ and does not overlap with the solid grains. We construct a finite grid and compute the diameter of the largest sphere at each grid point. The pore size distribution is evaluated via the cumulative histogram of the probability that a point in the pore volume belongs in a sphere with diameter $R$ greater than or equal of a pore width $s$.
The small angle scattering intensity $I(q)$ was computed from a digitized 3D image of the structure with a voxel size of $0.571 \mathrm{~nm}$, much smaller than the minimum particle diameter $3.78 \mathrm{~nm}$ and well below the box size $L=585.54$ $\mathrm{nm}$. The two point fluctuation autocorrelation function $\eta^{2}(r)$ was first evaluated and then $I(q)$ is given by

$$
I(q)=-\frac{2 \pi(\Delta \rho)^{2}}{q} \frac{d\left(\operatorname{Re}\left(\tilde{\eta}^{2}(q)\right)\right.}{d q}
$$

where $q$ is the wave vector, $\Delta \rho$ is the scattering length density contrast and $\tilde{\eta}^{2}(q)$, the 1D Fourier transform of $\eta^{2}(r)$ [11].

The pore chord length distribution is obtained by propagating lines with random direction from random origin points in order to accumulate the histogram of the lengths in the pore [12]. Chord length distributions are sensitive to the surface roughness and have been proven effective in characterising porous media [13].

\section{Results}

The out-of-equilibrium aggregation of the nano-grains results in heterogeneous morphology of the solid at the mesoscale. Capillary pores separate the strands of the dense network of polydisperse cement hydrates. Here, we characterise the structure of the solid and the pore of a nano-granular C-S-H configuration at hardened cement paste.

The total volume fraction $\eta$ of the system represents the fraction of the simulation box volume filled by spherical nano-grains. Figure 1 shows the local volume fraction distribution $\eta_{\text {local }}$ of cement hydrates of a configuration with $\eta=0.52$. The distribution displays a distorted Gaussian shape with maximum value at $\eta_{\text {local }}=0.68$. This maxima can be used to estimate the volume fraction of the capillary pores as $\eta / \eta_{\max }$. This is in agreement with $\mathrm{X}$-ray tomographic experiments [14]. According to Powers and Brownyard model [15] that links the volume of the capillary pores to the initial water $(\mathrm{w} / \mathrm{c})$ to cement ratio our configuration corresponds to a cement paste with $w / c \simeq 0.45$.

Figure 2 shows the distribution of the pore sizes in the same cement configuration. There are nanopores of 0-3 $\mathrm{nm}$, called gel pores in the cement literature, and mesopores of 3-30 nm, called capillary pores. The abundant amount of nanopores is the result of the local packing of grains. The larger pores have a broad distribution in sizes and result from the out-of-equilibrium aggregation of the nano-grains. The mesopores form a connected network which is crucial for the transport properties in cement. The pore size distribution of our configuration has all the characteristic reported by experiments of $N_{2}$ adsorption/desorption and NMR [1, 15-17].

SANS is a commonly used experimental technique to characterize the texture of hardened cement pastes $[7,18-$ 20]. Figure 3 shows the SANS data from [21] compared with the scattering intensity computed for our configuration. The two curves have a good agreement especially for most of the range of $q$ values. The plateau at low 


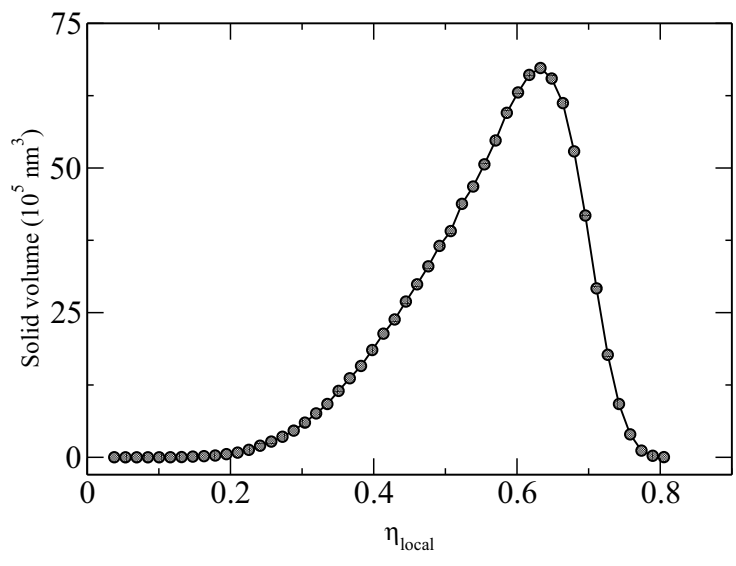

Figure 1: Local volume fraction distribution of hardened cement paste of total volume fraction $\eta=0.52$.

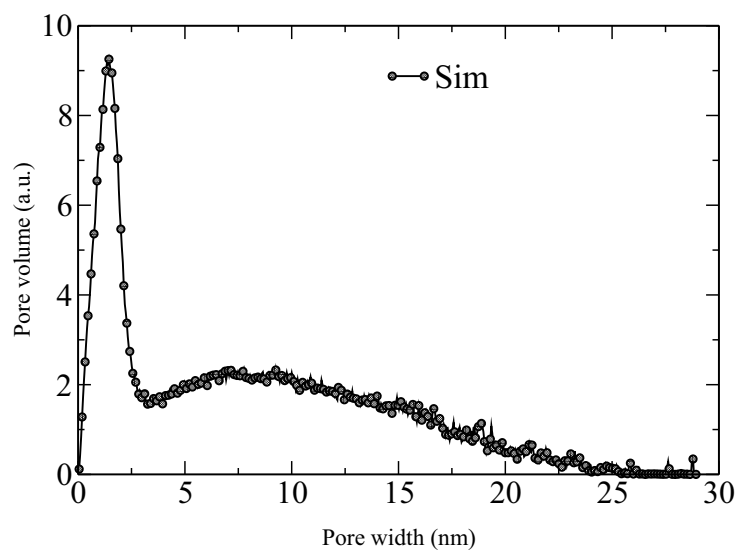

Figure 2: Pore size distribution of cement configurations of total volume fraction $\eta=0.52$.

wave vector $\mathrm{q}$, where the simulation data are restricted by the system size, is associated to a correlation length of about $\pi / q=40 \mathrm{~nm}$, ten times smaller than the box size $(585.54 \mathrm{~nm})$. The shift in the intensity axis arises from the fact that we compare simulation data of pure $\mathrm{C}$ S-H with experimental data of Ordinary Portland Cement (OPC) where other hydration products such as ettringite, portlandite etc. are present. At large $q$ values we find a dependence $I(q) \sim q^{-4}$ typical of a Porod regime [22] indicating that the nano-grain surfaces have a sub-nano metric roughness [23]. At smaller $q, I(q)$ follows $q^{-3}$ dependence for more than one order of magnitude in length as reported and discussed in the literature [6, 20, 24]. The low $q$ regime indicates long range spatial correlations and heterogeneities over lengths of $3-40 \mathrm{~nm}$ pointing on the crucial role of the mesopores at the same length range.

The mesoscale structure of cement hydrates was further characterized by chord lengths that are sensitive to surface roughness. The comparison of the pore chord length distribution for our sample with that obtained from SEM images of a neat OPC paste in [13] is shown in Fig. 4). The pixel dimension in the experiments is assumed to be of $\simeq 0.56 \mathrm{~nm}$. The comparison shows good agreement

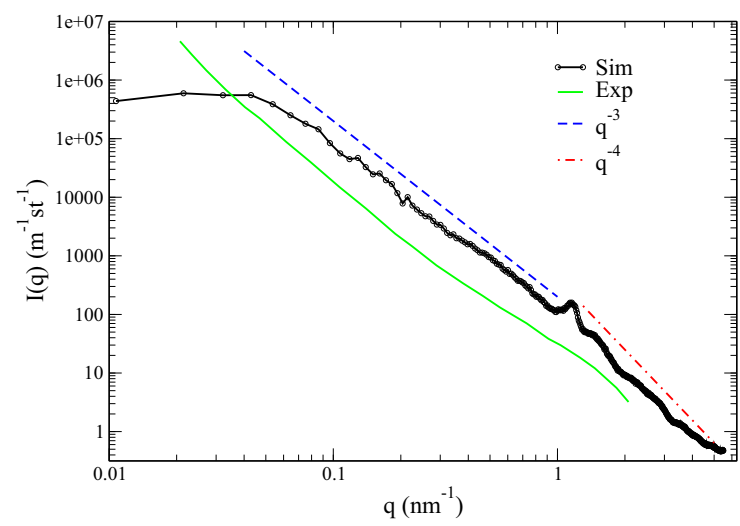

Figure 3: Scattering intensity computed from configurations of total volume fraction $\eta=0.52$ (Sim), compared with SANS data from [21]. The Porod regime $q^{-4}$ and $q^{-3}$ dependence are depicted by dashed lines.

until lengths of few tenths of $\mathrm{nm}$ and the limitation of the simulation system size.

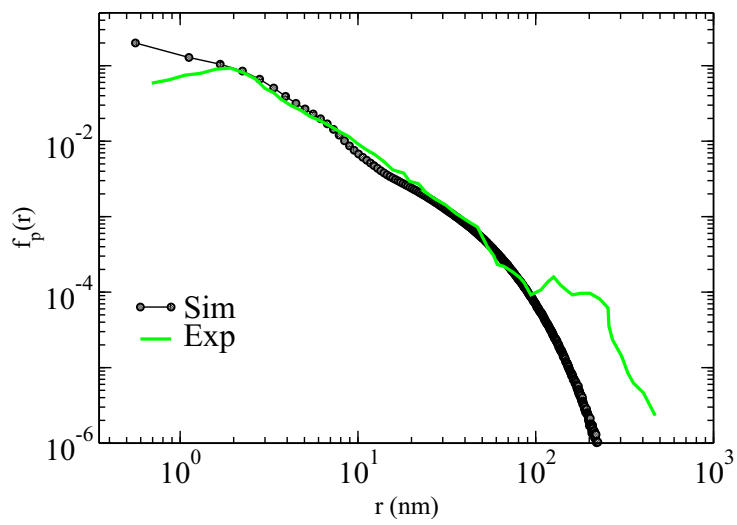

Figure 4: Pore chord length distribution of configurations of total volume fraction $\eta=0.52$ compared with the pore chord length distribution computed from SEM images of Ref. [13].

\section{Conclusions}

In this work, we present a nanogranular model for precipitation of cement hydrates that is able to capture the complex structure of solid and pore of hardened cement paste. Cement hydrates precipitate, aggregate and densify to form a solid network of a continuous distribution of packing fractions with a connected mesopore network. An extensive comparison of our simulated structure with experiments reveals the crucial role of structural heterogeneities that arises during precipitation of the nanograins.

Moreover, variations of the local density in hardened cement pastes have been reported in nanoindentation experiments [25] with most of the experiments reporting values $>0.55$ for local packing as nanoindentation cannot detect the softer areas. Here we find that areas of even lower 
packing fraction coexist with the denser ones. However in agreement with nanoindentation experiments the percolated dense areas carry the mechanical load [26].

Finally we note that our model is also able to reproduce the mechanical response of cement as reported by nanoindentation experiments [26]. The realistic description of both structure and mechanics of cement hydrates in our nano-granular model provides a model for investigating degradation mechanisms in cement such as fracture, creep, freeze-thawing etc.

\section{Acknowledgements}

This work is supported by Schlumberger under the XCEM project and by the CSHub at MIT. EDG acknowledges support from SNSF (Grant No. PP00P2_150738) and the National Science Foundation (Grant No. NSF PHY1125915). This work has been carried out within the framework of the ICoME2 Labex (ANR-11-LABX-0053) and the A*MIDEX projects (ANR-11-IDEX-0001-02) cofunded by the French program "Investissements d'Avenir" which is managed by the ANR, the French National Research Agency.

\section{References}

[1] H.F. Taylor, Cement chemistry (Thomas Telford, 1997)

[2] R.J.M. Pellenq, A. Kushima, R. Shahsavari, K.J. Van Vliet, M.J. Buehler, S. Yip, F.J. Ulm, Proc Natl Acad Sci USA. 106, 16102 (2009)

[3] D. Lootens, P. Hébraud, E. Lécolier, H. Van Damme, Oil Gas Sci. Technol. 59, 31 (2004)

[4] K. Ioannidou, R.J.M. Pellenq, E. Del Gado, Soft Matter 10, 1121 (2014)

[5] K. Ioannidou, M. Kanduc, L. Li, D. Frenkel, J. Dobnikar, E. Del Gado, Nature Communications 7, 12106 (2016)

[6] A.J. Allen, J.J. Thomas, H.M. Jennings, Nat Mater 6, 311 (2007)

[7] W.S. Chiang, E. Fratini, P. Baglioni, D. Liu, S.H. Chen, J. Phys. Chem. C 116, 5055 (2012)
[8] R.J.M. Pellenq, H. Van Damme, MRS Bull 29, 319 (2004)

[9] E. Del Gado, K. Ioannidou, E. Masoero, A. Baronnet, R.M. Pellenq, F.J. Ulm, S. Yip, Eur Phys J-Spec Top 223, 2285 (2014)

[10] S. Bhattacharya, K.E. Gubbins, Langmuir 22, 7726 (2006)

[11] P. Levitz, D. Tchoubar, J Physique I 2, 771 (1992)

[12] J. Mering, D. Tchoubar, J Appl Crystallogr 1, 153 (1968)

[13] P. Levitz, Adv Colloid Interface Sci 76, 71 (1998)

[14] J.C. da Silva, P. Trtik, A. Diaz, M. Holler, M. GuizarSicairos, J.r. Raabe, O. Bunk, A. Menzel, Langmuir 31, 3779 (2015)

[15] T. Powers, T. Brownyard, PCA Bulletin 22 (1948)

[16] R.S. Mikhail, L.E. Copeland, S. Brunauer, Can J Chem 42, 426 (1964)

[17] R. Valckenborg, L. Pel, K. Kopinga, J Phys D: Appl Phys 35, 249 (2002)

[18] A.J. Allen, J.C. McLaughlin, D.A. Neumann, R.A. Livingston, J Mater Res 19, 3242 (2004)

[19] E. Fratini, A. Faraone, F. Ridi, S.H. Chen, P. Baglioni, J Phys Chem C 117, 7358 (2013)

[20] S. Bae, R. Taylor, D. Shapiro, P. Denes, J. Joseph, R. Celestre, S. Marchesini, H. Padmore, T. Tyliszczak, T. Warwick et al., J Am Ceram Soc 98, 4090- (2015)

[21] K.J. Krakowiak, J.J. Thomas, S. Musso, S. James, A.T. Akono, F.J. Ulm, Cem Concr Res 67, 103 (2015)

[22] A. Guinier, G. Fournet, Small-Angle Scattering of X-rays (Wiley, 1955)

[23] S. Brisard, P. Levitz, Phys Rev E 87, 013305 (2013)

[24] S. Brisard, R.S. Chae, I. Bihannic, L. Michot, P. Guttmann, J. Thieme, G. Schneider, P.J. Monteiro, P. Levitz, Am Mineral 97, 480 (2012)

[25] G. Constantinides, F.J. Ulm, J Mech Phys Solids 55, 64 (2007)

[26] K. Ioannidou, K.J. Krakowiak, M. Bauchy, C.G. Hoover, E. Masoero, S. Yip, F.J. Ulm, P. Levitz, R.J.M. Pellenq, E. Del Gado, Proceedings of the National Academy of Sciences 113, 2029 (2016) 